\section{The investigation of sleep disordered breathing: seeing through a glass, darkly?}

\author{
Catherine M Hill, ${ }^{1}$ Hazel J Evans ${ }^{2}$
}

Timely diagnosis and treatment of obstructive sleep apnoea (OSA) in childhood is important to prevent morbidity and increased healthcare utilisation. ${ }^{1}$ In this issue, Burke et $a l^{2}$ highlight an important clinical question-how to best diagnose OSA in children, asking the question: is one night of oximetry enough? They note the limited availability of polysomnography, the international gold standard diagnostic test ${ }^{3}$ for OSA and that pulse oximetry is widely available.

However, widespread availability of oximetry risks widespread misinterpretation. It is crucial to understand that not all oximeters are 'born equal' and the technology available may have significant limitations. The diagnostic yield of any oximeter will depend crucially on the device used and its settings, the scoring criteria applied to the trace, alongside the clinical interpretation of the data. Modern oximeters are able to detect and remove motion artefact, which is critical in restless young children (figure 1). Oximeters need to be set with short averaging times (usually maximum $3 \mathrm{~s}$ ) to avoid smoothing out of brief desaturation events (figure 2). McGill scoring criteria are recommended with a score $>1$ (three or more clusters of desaturation events $\geq 4 \%$ and at least three desaturations to $<90 \%$ ) being indicative of OSA, ${ }^{3}$ but as noted by Burke and colleagues, the risk of false negative results is high.

Clinical interpretation of oximetry requires a thorough understanding of sleep physiology and how this changes with age. Children are most likely to obstruct their upper airway in rapid eye movement (REM) sleep, when skeletal muscle atonia causes relaxation and narrowing of the pharyngeal airway. REM sleep is not evenly dispersed through the night. Four hours of data collected in the early part of

${ }^{1}$ CES division, Faculty of Medicine, Southampton Children's Hospital, Southamton, UK; ${ }^{2}$ Department of Respiratory Medicine, Southampton Children's Hospital, Southampton, UK

Correspondence to Dr CES division, Faculty of Medicine, Southampton Children's Hospital, Southamton, UK; cmh2@soton.ac.uk the night may capture very little REM sleep, conversely 4 hours of data at the end of the night may contain a disproportionate amount of REM sleep. Knowledge of sleep architecture is needed to interpret studies intelligently. Skilled clinicians can learn to recognise likely REM sleep due to the characteristic increase in heart rate variability and can make a judgement on whether or not the oximetry study contained REM sleep periods. However, a fundamental limitation of oximetry is that it is impossible to be certain that a child is awake or asleep. 'Sleep studies' may in fact be 'wake studies'. A further limitation is that not all obstructive upper airway events in sleep are associated with oxygen desaturation, in some cases children arouse from sleep before desaturation occurs. Nonetheless, these arousal related events can be clinically significant causing fragmentation of sleep and daytime cognitive and behavioural difficulties. ${ }^{3}$

Finally, clinical context is key, and oximetry must be interpreted with caution in children with Down syndrome and central nervous system pathology when oxyhaemoglobin desaturation may reflect central, as well as, obstructive apnoeic episodes. Distinguishing central apnoea from obstructive apnoea is only possible when respiratory effort is

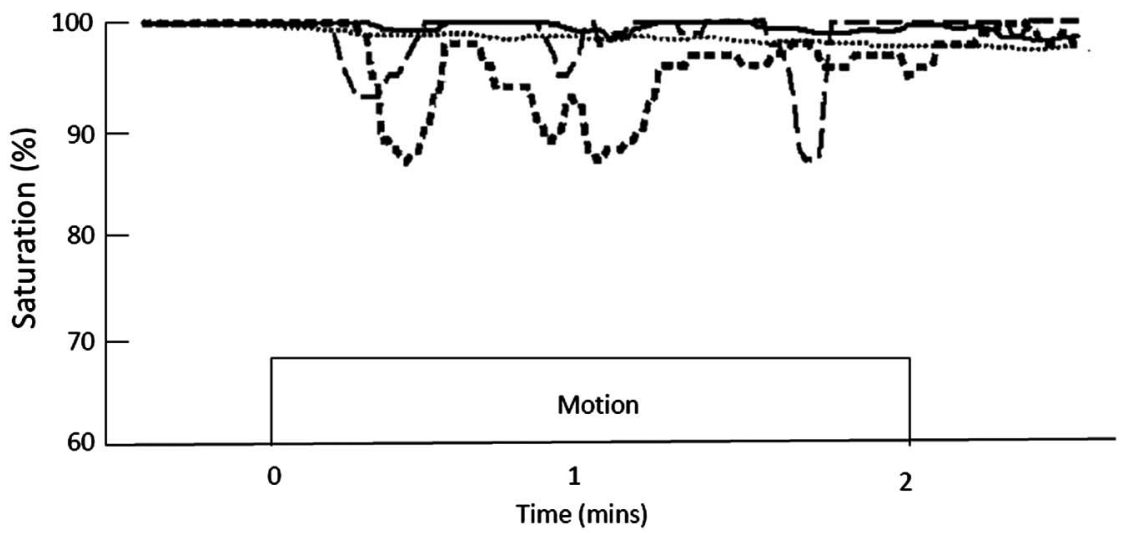

.......... $\mathrm{SpO}_{2}$ (control) — Masimo SET $\quad \ldots .$. Nellcor $\mathrm{N}-395 \quad$ - - Agilent Virdia $24 \mathrm{C}$

Figure 1 Influence of oximeter device on motion artefact. Graphs of $\mathrm{SpO}_{2}$ measured by three different oximeters during hand motion with subject breathing room air. False desaturations displayed by two instruments (hashed lines) compared with motion-resistant device (solid line). Reproduced with permission of the publisher Wolters Kluwer Health, Inc, from Barker. ${ }^{6}$ 


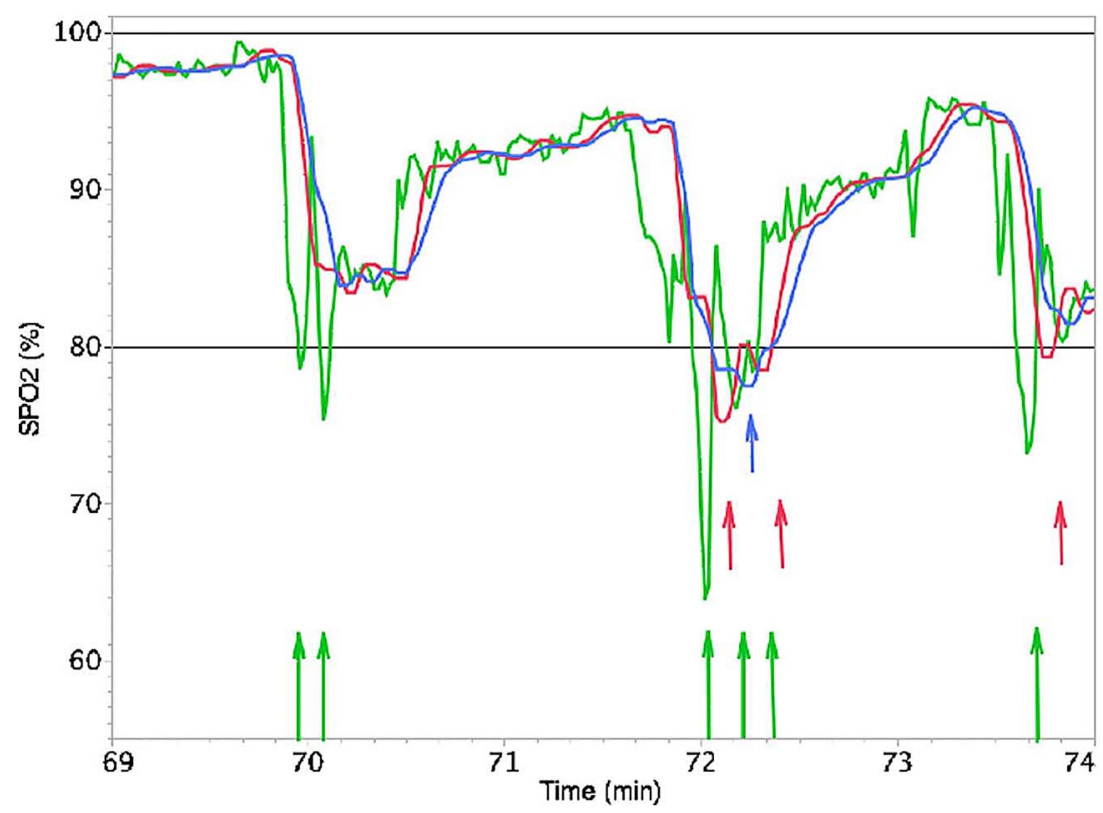

Figure 2 Influence of the averaging time on the number of desaturations For an alarm threshold at $80 \% \mathrm{SpO}_{2}$ (straight line), an averaging time of $3 \mathrm{~s}$ (green) results in six desaturations, while an averaging time of 10 (red) or $16 \mathrm{~s}$ (blue) results in three and one desaturation(s), respectively. Reproduced from Vagedes et al. ${ }^{7}$

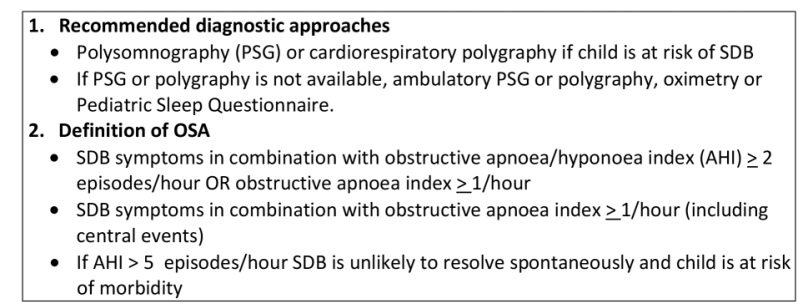

Figure 3 European Task Force: objective diagnosis and assessment of SDB severity. ${ }^{3}$ OSA, obstructive sleep apnoea; SDB, sleep disordered breathing.

diagnose OSA could more usefully be reframed as 'is one night of oximetry too much..... without expert interpretation!' Oximetry as a diagnostic option for OSA is acknowledged by the European Respiratory Society Task Force ${ }^{3}$ as sometimes necessary in 'resource limited settings', but importantly, not as the default diagnostic approach. If oximetry is used, the question about night to night variability in respiratory events is an important interpretation of, oximetry studies as part of an investigative pathway for sleep disordered breathing is lacking and should be developed. In the interim, we advocate that the recommendations from the European Task Force ${ }^{3}$ are widely adopted (figure 3) and that cardiorespiratory polygraphy services are further developed to meet the resource gap where full polysomnography facilities are absent.

Competing interests None declared.

Provenance and peer review Commissioned; internally peer reviewed.

To cite Hill CM, Evans HJ. Arch Dis Child 2016;101:1082-1083.

Received 3 March 2016

Accepted 5 August 2016

Published Online First 1 September 2016

\section{Linked}

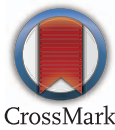

http://dx.doi.org/10.1136/archdischild-2015-308981

Arch Dis Child 2016:101:1082-1083. doi:10.1136/archdischild-2016-310483

\section{REFERENCES}

1 Tarasiuk A, Greenberg-Dotan S, Simon-Tuval T, et al. Elevated morbidity and health care use in children with obstructive sleep apnea syndrome. Am J Respir Crit Care Med 2007;175:55-61.

2 Burke RM, Maxwell B, Hunter C, et al. Night-to-night variation of pulse oximetry in children with sleepdisordered breathing. Arch Dis Child 2016;101:1095-9.

3 Kaditis AG, Alonso Alvarez ML, Boudewyns A, et al. Obstructive sleep disordered breathing in 2- to 18-year-old children: diagnosis and management. Eur Respir J 2016;47:69-94.

4 Terrill PI, Dakin C, Hughes I, et al. Nocturnal oxygen saturation profiles of healthy term infants. Arch Dis Child 2015;100:18-23.

5 Alonso-Álvarez M, Terán-Santos J, Ordax Carbajo E, et al. Reliability of home respiratory polygraphy for the diagnosis of sleep apnea in children. Chest 2015;147:1020-8.

6 Barker SJ. "Motion-Resistant" pulse oximetry: a comparison of new and old models. Anesth Analg 2002;95:967-72.

7 Vagedes J, Bialkowski A, Wiechers C, et al. A conversion formula for comparing pulse oximeter desaturation rates obtained with different averaging times. PLOS ONE 2014;9:e87280. 